\title{
Vancomycin use in a large teaching hospital in Shiraz, Islamic Republic of Iran, 2003
}

M. Askarian, ${ }^{1}$ O. Assadian, ${ }^{2}$ GhR. Safaee, ${ }^{3}$ A. Golkar, ${ }^{3}$ S. Namazi ${ }^{4}$ and M.R. Movahed ${ }^{5}$

استعمال الفانكوميسين في مستشفى تعليهي كبير في شيراز بجمهورية إيران الإسلامية، عام 2003

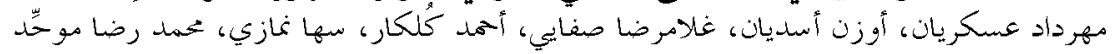

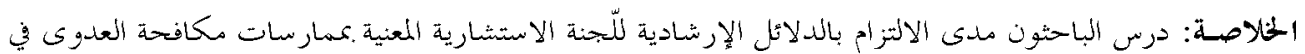

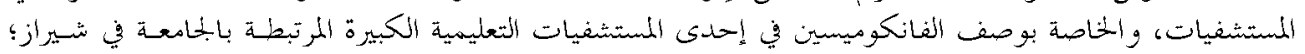

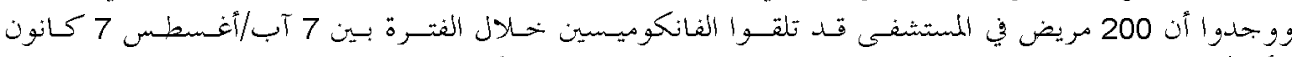

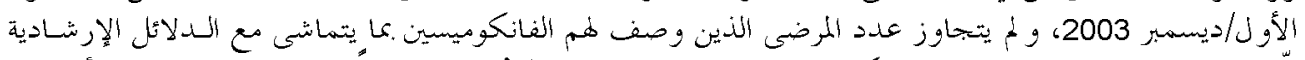

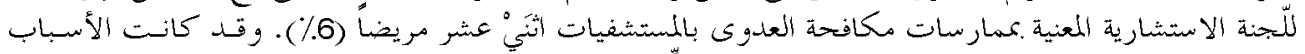

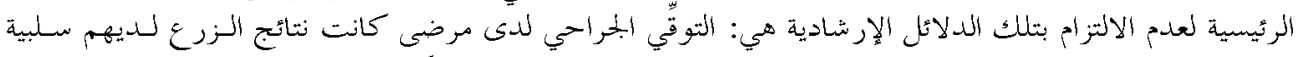

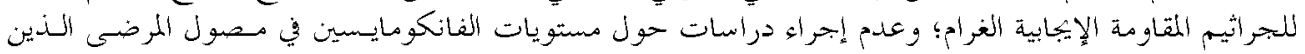

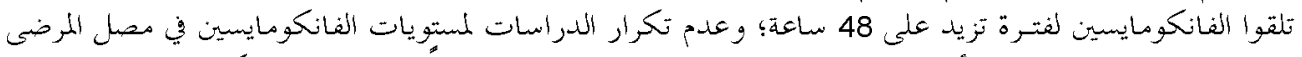

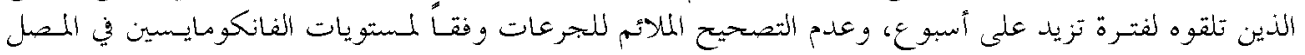

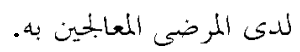

ABSTRACT We investigated adherence to the Hospital Infection Control Practice Advisory Committee (HICPAC) guidelines on vancomycin prescription in a large university-affiliated hospital in Shiraz. From August to December 2003, 200 hospitalized patients received vancomycin. For only $12(6 \%)$ of these patients was vancomycin prescribed appropriately according to HICPAC guidelines. The main reasons why vancomycin use did not comply with HICPAC recommendations were: surgical prophylaxis in patients with negative cultures for resistant Gram-positive organisms, no investigation of vancomycin serum levels in patients receiving $>48$ hours of vancomycin, vancomycin serum levels not repeated in patients receiving $>1$ week of vancomycin, no appropriate adjustment of dosage with respect to serum levels in patients receiving vancomycin.

L'utilisation de la vancomycine en 2003 au sein d'un grand hôpital universitaire à Chiraz en République islamique d'Iran

RÉSUMÉ Dans un grand hôpital universitaire de Chiraz, nous avons évalué l'adhésion aux directives du HICPAC (pour Hospital Infection Control Practices Advisory Committee en matière de prescription de la vancomycine. Entre août et décembre 2003, 200 patients hospitalisés ont reçu de la vancomycine. La vancomycine n'a été prescrite dans le respect des directives du HICPAC que chez 12 (6\%) de ces patients. Les principales causes de la non-conformité de la prescription de la vancomycine aux recommandations du HICPAC sont les suivantes : prophylaxie chirurgicale en présence de cultures négatives de germes Gram-positif résistants, non-détermination des concentrations sériques de vancomycine en cas de traitement d'une durée supérieure à 48 heures, dosage de la vancomycine sérique non répété chez les patients traités avec la vancomycine pendant plus d'une semaine et absence d'adaptation correcte de la posologie en fonction des concentrations sériques chez les patients sous vancomycine.

${ }^{1}$ Department of Community Medicine; ${ }^{4}$ Department of Pharmacy, Shiraz University of Medical Sciences, Shiraz, Islamic Republic of Iran (Correspondence to M. Askarian: askariam@sums.ac.ir).

2University Hospital Vienna, Clinical Institute for Hygiene and Medical Microbiology, Medical University of Vienna, Vienna, Austria.

${ }^{3}$ Jahrom Medical School, Jahrom, Islamic Republic of Iran.

${ }^{5}$ Department of Medicine, University of California, Irvine Medical Center, California, United States of America. Received: 07/08/05; accepted: 31/10/05 


\section{Introduction}

Recent studies have shown inappropriate use of antimicrobial agents, which is one of the most important factors in the development of resistant pathogens [1-3].

Vancomycin is a core antimicrobial agent in the treatment of infections caused by Gram-positive pathogens. Exposure to this antibiotic has been shown to be a significant risk factor for colonization and infection with vancomycin-resistant enterococci (VRE), and appropriate use of vancomycin is important in order to prevent the emergence of VRE and the possible spread of VRE genes to other bacteria [4-7].

Since the first report of VRE in 1998, this organism has become a major health problem in hospitals in North America and Europe [8]. In recent reports of the National Nosocomial Infection Surveillance System, the pooled mean prevalence of VRE was $12.7 \%$ in intensive care units in the United States [9].

The Hospital Infection Control Practice Advisory Committee (HICPAC) of the Centers for Disease Control and Prevention (CDC) has issued guidelines to prevent the spread of VRE and it also recommends the judicious use of vancomycin [10].

To date, no data are available on vancomycin use by Iranian physicians and their adherence to HICPAC guidelines. Therefore, we investigated the adherence to these guidelines in a large university-affiliated hospital in the southern part of the Islamic Republic of Iran.

\section{Methods}

The hospital "A" with 750 beds is located in Shiraz, the largest city in the southern part of the Islamic Republic of Iran. It is a referral centre for one-quarter of the country's medical cases with about 21000 admissions per year and an average bed occupancy rate of $75 \%$.

This was a cross-sectional study conducted from 15 August to 16 December, 2003. On a daily basis, the hospital records of all patients on all wards who received vancomycin were reviewed and the reasons for prescribing this antibiotic were compared with the HICPAC recommendations. Data were collected using a questionnaire, which included patient's age, sex, date of admission, length of hospitalization, date of urinary catheterization (if applicable), duration and type of central venous access and endotracheal intubation, and duration of vancomycin therapy. The judgement on whether vancomycin prescription was empirical or based on culture, treatment or prophylaxis was strictly based on HICPAC recommendations. Criteria for evaluation of appropriate use of vancomycin are summarized in Table 1.

\section{Results}

Table 2 give information on the patients and vancomycin therapy. During the study period, 200 hospitalized patients received vancomycin; 119 males (59.5\%) and 81 females. Mean age and standards deviation (SD) of the patients was 25 (SD 24) years (range: 1 month to 80 years). Mean length of stay was 23 (SD 14) days (range: 1 to 98 days). Total mean duration of vancomycin therapy and mean duration of hospitalization before the first dose of vancomycin was 15 (SD 14) days (range: 1 to 37 days) and 5 (SD 6) days (range: 0 to 34 days) respectively.

No patient was allergic to $\beta$-lactam antimicrobials. Prosthetic devices were present in $33.3 \%$ of the patients including urinary catheters $(8 \%)$, central venous lines $(7 \%)$ and mechanical ventilation $(1.5 \%)$, with a total duration of each being 117 urinary 
Table 1 Criteria for evaluation of appropriate use of vancomycin used in this study (based on [10])

Situations in which the use of vancomycin is appropriate or acceptable.

- For treatment of serious infections caused by beta-lactam-resistant Gram-positive microorganisms.

- For treatment of infections caused by Gram-positive micro-organisms in patients who have serious allergies to beta-lactam antimicrobials.

- When antibiotic-associated colitis fails to respond to metronidazole therapy or is severe and potentially life-threatening.

- $\quad$ Prophylaxis, as recommended by the American Heart Association, for endocarditis following certain procedures in patients at high risk for endocarditis.

- $\quad$ Prophylaxis for major surgical procedures involving implantation of prosthetic materials or devices (e.g. cardiac and vascular procedures and total hip replacement) at institutions that have a high rate of infections caused by MRSA or methicillin-resistant S. epidermidis.

- A single dose of vancomycin administered immediately before surgery is sufficient unless the procedure lasts $>6$ hours, in which case the dose should be repeated. Prophylaxis should be discontinued after a maximum of 2 doses.

Situations in which the use of vancomycin should be discouraged:

- $\quad$ Routine surgical prophylaxis other than in a patient who has a life-threatening allergy to beta-lactam antibiotics.

- Empirical antimicrobial therapy for a febrile neutropenic patient, unless initial evidence indicates that the patient has an infection caused by Gram-positive micro-organisms and the prevalence of infections caused by MRSA in the hospital is substantial.

- Treatment in response to a single blood culture positive for coagulase-negative staphylococcus, if other blood cultures taken during the same time frame are negative (i.e. if contamination of the blood culture is likely).

- $\quad$ Continued empirical use for presumed infections in patients whose cultures are negative for beta-lactam-resistant Gram-positive micro-organisms.

- $\quad$ Systemic or local prophylaxis for infection or colonization of in-dwelling central or peripheral intravascular catheters.

- Selective decontamination of the digestive tract.

- Eradication of MRSA colonization.

- Primary treatment of antibiotic-associated colitis.

- $\quad$ Routine prophylaxis for very low-birth weight infants (<1500 g).

- Routine prophylaxis for patients on continuous ambulatory peritoneal dialysis or haemodialysis.

- Treatment (chosen for dosing convenience) of infections caused by beta-lactam-sensitive Gram-positive micro-organisms in patients who have renal failure.

- Use of vancomycin solution for topical application or irrigation.

Inappropriate practice for continued use of vancomycin:

- Prophylaxis infusion not completed within 30 minutes of incision.

- Prophylaxis continued for $>1$ dose if the surgical procedure is $<6$ hours duration and $>2$ doses if the surgical procedure is $>6$ hours duration.

- $\quad$ Lack of vancomycin serum levels in patients receiving $>48$ hours of vancomycin therapy.

- Vancomycin serum levels not repeated in patients receiving $>1$ week of vancomycin therapy.

- Dosage not adjusted appropriately with respect to serum levels in patients receiving vancomycin therapeutically (acceptable serum levels are a trough of $5-10 \mathrm{~g} / \mathrm{mL}$ and a peak of $20-35 \mathrm{~g} / \mathrm{mL})$. 
Table 2 Information on patient's prescribed vancomycin and evaluation of its use

\begin{tabular}{lcc}
\hline Variable & No. & $\%$ \\
& $(n=200)$ & \\
\hline
\end{tabular}

Age of patient (years)

$<1$

$1-9$

$10-19$

20-29

$30-39$

$40-49$

$50-59$

$60-69$

$>70$

Sex

Male

Female

Duration of hospitalization

(days)

$<10$

$10-19$

20-29

30-39

40-49

50-59

$>60$

Method of administration of vancomycin

Intravenous

Patient received vancomycin appropriately

Yes

No

Age of patients (years)

(range 1 month-80)

Total duration of

hospitalization (days)

(range 1-98)

Duration of hospitalization

before first dose of

vancomycin (days)

(range 0-34)

Duration of vancomycin

therapy (days) (range 1-37)

Total $g$ of vancomycin used

$S D=$ standard deviation. catheter days, 24 central venous line days, and 60 person days of ventilation.

In $54 \%$ of the patients, vancomycin was prescribed for infections. The total amount of vancomycin prescribed was $4374 \mathrm{~g}$ corresponding to 2187 defined daily doses (DDD). The total observed standardized vancomycin usage was 48.9 DDDs/100 patient days. Of the 200 patients, $92 \%$ were prescribed the appropriate vancomycin dose and $51.5 \%$ received the appropriate duration of treatment. However, only in 12 $(6.0 \%)$ of the patients was the prescription of vancomycin appropriate according to HICPAC guidelines.

The main reasons why the use of vancomycin did not comply with HICPAC recommendations were: continued empirical use for surgical prophylaxis in patients whose cultures were negative for resistant Gram-positive organisms (100\%), no investigation of vancomycin serum levels in patients receiving $>48$ hours of vancomycin therapy $(100 \%)$, vancomycin serum levels not repeated in patients receiving $>1$ week of vancomycin therapy $(100 \%)$, no appropriate adjustment of dosage with respect to serum levels in patients receiving vancomycin $(100 \%)$, continued empirical use of vancomycin for presumed infections in patients whose cultures were negative for beta-lactam-resistant Gram-positive microorganisms $(66 \%)$, treatment of infections caused by beta-lactam-sensitive Grampositive micro-organisms in patients with renal failure $(8 \%)$, routine prophylaxis for very low-birth weight infants (infants < $1500 \mathrm{~g})(2.5 \%)$, treatment in response to a single blood culture positive for coagulasenegative staphylococci although other cultures taken during the same time frame were negative $(0.5 \%)$, eradication of methicillinresistant Staphylococcus aureus (MRSA) colonization $(0.5 \%)$, primary treatment of antibiotic-associated colitis $(0.5 \%)$, routine 
prophylaxis for patients on haemodialysis $(0.5 \%)$, and use of vancomycin solution for topical application or irrigation $(0.5 \%)$.

\section{Discussion}

Infectious diseases are one of the greatest causes of morbidity and mortality and the spread of multi-resistant organisms is playing a significant part in this. The excessive and inappropriate use of antimicrobial agents remains one of the most important factors inducing resistance [1]. Previous studies have shown that up to $50 \%$ of prescribing may be inappropriate [11].

The gravity of the problem can be seen by the many strategies that have been reported to improve antimicrobial prescribing in hospitals, including educational programmes $[3-5,10]$, development of restrictive hospital formulae $[4,6,12]$, limitation on reports of sensitivity tests, regulation of interaction between pharmaceutical representatives and physicians, automatic stop orders at 72 hours $[5,6,13]$, written justification for specific antimicrobial agents and/or requirement for expert approval before or after prescribing $[5,12,13]$ and implementation of computerized guidelines [14].

Increased prescribing of vancomycin in the past decade has been linked to the development and spread of VRE [2,4]. This finding has led to efforts to reduce total vancomycin use by decreasing inappropriate usage. However, appropriate antimicrobial use is often subjective and difficult to define. Specific methods to standardize the evaluation of antimicrobial use have not yet been developed [1]. HICPAC recommendations give specific guidelines on what constitutes appropriate vancomycin use, listing 5 situations in which vancomycin use is deemed appropriate and another 12 in which it is not [10].
Before publication of the HICPAC guidelines, vancomycin use had increased substantially; one university hospital had documented a 20-fold increase from 1981 to $1991[4,15,16]$.

More than $90 \%$ of the vancomycin orders evaluated in our hospital were inconsistent with the HICPAC guidelines. Moreover, most of the inappropriate vancomycin use in our study was complicated by the absence of therapeutic monitoring to ensure adequate non-toxic doses. This is higher than rates reported by other researchers which range from $24 \%$ to $65 \%[4,17,18]$. However, these outcomes were predictable because few of the physicians in our hospital were aware of the HICPAC recommendations.

While the initial administration of vancomycin could be justified on the basis of empirical therapy, there was a subsequent failure to provide appropriate revision of treatment in $66 \%$ of patients. This is similar to other published studies and could be corrected with an antibiotics stop order at 72 hours after initiation of therapy $[5,17]$. Close cooperation between physician and pharmacists seems to be essential. Lipsky et al. and Singer et al. reported that surgical prophylaxis was identified as the major source of inappropriate use of vancomycin in their studies [4,5]. However, we found only 1 case of inappropriate vancomycin use for surgical prophylaxis.

A subset of our findings demonstrated that $85 \%$ of haematology/oncology patients and $28 \%$ of all patients received vancomycin as the initial therapy for febrile neutropenia. Again, this is a higher rate than in other reports [19] and such therapy is not necessary because it has been reported that vancomycin usage can be withheld following initial $\beta$-lactam aminoglycoside therapy for up to 72 hours to determine whether resolution of fever occurs or not [20]. 
Vancomycin use in our hospital is high and generally inappropriate. We believe however that it could be controlled with a 3-day stop order on its prescription to ensure careful assessment of its ongoing need and by educating our haematologists/ oncologists to use vancomycin only if fever does not settle after 72 hours of initial $\beta$ lactam aminoglycoside therapy. Education of physicians in general and awareness of the HICPAC recommendations could also help to reduce the inappropriate use of this medicine.

\section{Acknowledgement}

This study was funded by the Deputy for Research at the Shiraz University of Medical Sciences (grant no. 82-1860).

\section{References}

1. Niederman MS. Appropriate use of antimicrobial agents: Challenges and strategies for improvement. Critical care medicine, 2003, 31:608-16.

2. Zaas AK e t al. Risk factors for development of vancomycin resistant enterococcal bloodstream infection in patients with cancer who are colonized with vancomycin-resistant enterococci. Clinical infectious disease, 2002, 35:1139-46.

3. Shin JW et al. Sudden increase of vancomycin-resistant enterococcal infections in a Korean tertiary care hospital: possible consequences of increased use of oral vancomycin. Journal of infection and chemotherapy, 2003, 9:62-7.

4. Lipsky BA et al. Improving the appropriateness of vancomycin use by sequential interventions. American journal of infection control, 1999, 27:84-91.

5. Singer MV et al. Vancomycin control measures at a tertiary-care hospital: Impact of interventions on volume and patterns of use. Infection control and hospital epidemiology, 1998, 19:248-53.

6. Shaikh $\mathrm{ZH}$ et al. Effectiveness of a multifaceted infection control policy in reducing vancomycin usage and vancomycinresistant enterococci at a tertiary care cancer centre. Journal of hospital infection, 2002, 51:52-8.

7. Fridkin SK et al. The effect of vancomycin and third-generation cephalosporins on prevalence of vancomycin-resistant enterococci in 126 U.S. adult intensive care units. Annals of internal medicine, 2001, 135:175-83.

8. Floret $\mathrm{N}$ et al. Evaluation of vancomycin use in a large university-affiliated hospital in eastern France in 1999. Pharmacy world \& science, 2001, 23:93-7.

9. NNIS System. National Nosocomial Infections Surveillance (NNIS) System Report, data summary from January 1992 through June 2003, issued August 2003. American journal of infection control, 2003, 31:481-98.

10. Recommendations for preventing the spread of vancomycin resistance. Hospital Infection Control Practices Advisory Committee (HICPAC). Infection control and hospital epidemiology, 1995, 16:10513.

11. Thuong $\mathrm{M}$ et al. Appropriate use of restricted antimicrobial agents in hospitals: the importance of empirical therapy and assisted re-evaluation. Journal of antimicrobial chemotherapy, 2000, 46:501-8.

12. Anglim AM et al. Effect of a vancomycin restriction policy on ordering practices during an outbreak of vancomycin-resistant Enterococcus faecium. Archives of internal medicine, 1997, 157:1132-6.

13. Keyserling $\mathrm{HL}$ et al. Vancomycin use in hospitalized pediatric patients. Pediatrics, 2003, 112:e104-11. 
14. Shojania KG et al. Reducing vancomycin use utilizing a computer guideline: results of a randomized controlled trial. Journal of the American Medical Informatics Association, 1998, 5:554-62.

15. Jarvis WR. Epidemiology, appropriateness, and cost of vancomycin use. Clinical infectious diseases, 1998, 26:1200-03.

16. Drori-Zeides $T$ et al. Practical guidelines for vancomycin usage, with prospective drug-utilization evaluation. Infection control and hospital epidemiology, 2000, 21:45-7.

17. De Castro MS et al. Evidence of inappropriate use of vancomycin in a university affiliated hospital in Brazil. Pharmacoepi- demiology and drug safety, 1999, 8:40511.

18. Logsdon BA et al. Evaluation of vancomycin use in a pediatric teaching hospital based on CDC criteria. Infection control and hospital epidemiology, 1997, 18:7802.

19. Ena $\mathrm{J}$ et al. The epidemiology of intravenous vancomycin usage in a university hospital. A 10-year study. Journal of the American Medical Association, 1993, 269:598-602.

20. Hopkins HA e tal. Vancomycin use in pediatric hematology-oncology patients. Infection control and hospital epidemiology, 2000, 21:48-50.

\section{Rational use of medicines}

Irrational use of medicines is a major problem worldwide. WHO estimates that more than half of all medicines are prescribed, dispensed or sold inappropriately, and that half of all patients fail to take them correctly. The overuse, underuse or misuse of medicines results in wastage of scarce resources and widespread health hazards. Examples of irrational use of medicines include: use of too many medicines per patient ("poly-pharmacy"); inappropriate use of antimicrobials, often in inadequate dosage, for non-bacterial infections; over-use of injections when oral formulations would be more appropriate; failure to prescribe in accordance with clinical guidelines; inappropriate selfmedication, often of prescription-only medicines; non-adherence to dosing regimes.

More information about WHO's work on selection and rational use of medicines can be found at: http://www.who.int/medicines/areas/rational_use/en/index.html 